\title{
Engineering Highly Homogenous Tetravalent IgGs with Enhanced Sperm Agglutination Potency
}

Bhawana Shrestha ${ }^{a}$, Alison Schaefer ${ }^{b}$, Elizabeth C. Chavez ${ }^{c}$, Alexander J. Kopp ${ }^{d}$, Timothy M. Jacobs ${ }^{e}$,

Thomas R. Moench ${ }^{f}$, and Samuel K. Lai ${ }^{a, b, e, f, *}$

${ }^{a}$ Department of Microbiology \& Immunology; ${ }^{b}$ UNC/NCSU Joint Department of Biomedical Engineering;

${ }^{c}$ Department of Biology; ${ }^{d}$ Department of Health Policy and Management; ${ }^{e}$ Division of Pharmacoengineering and Molecular Pharmaceutics; University of North Carolina at Chapel Hill, Chapel Hill, NC 27599, USA; fMucommune, LLC., Durham, NC 27709, USA

\section{Corresponding Author}

Samuel K. Lai

Division of Pharmacoengineering and Molecular Pharmaceutics

University of North Carolina at Chapel Hill

Marsico Hall 4213, 125 Mason Farm Road

Chapel Hill, NC 27599

Email: lai@unc.edu 
Abstract: Millions of women avoid using available contraceptives and risk unintended pregnancies every year, due to perceived and/or real side-effects associated with the use of exogenous hormones. Naturally occurring anti-sperm antibodies can prevent fertilization in immune infertile women by limiting sperm permeation through mucus, particularly multivalent antibodies such as slgA that offers robust agglutination potencies. Unfortunately, slgA remains challenging to produce in large quantities and easily aggregates. Here, we designed two tetravalent anti-sperm IgGs with a Fab domain previously isolated from an immune infertile woman. Both constructs possess at least 4-fold greater agglutination potency and induced much more rapid sperm agglutination than the parent IgG while exhibiting comparable production yields and identical thermostability as the parent IgG. These tetravalent IgGs offer promise for non-hormonal contraception and underscore the multimerization of $\lg G$ as a promising strategy to improve existing mAb therapeutics.

Globally, over $40 \%$ of all pregnancies are unintended, which creates an enormous burden on healthcare systems.[1] For instance, in the U.S., nearly half of all pregnancies are unintended, resulting in an annual burden in excess of $\$ 20$ billion per year.[2,3] Despite the availability of cheap and effective contraceptive methods, many women are dissatisfied with available contraceptive methods, particularly due to real and/or perceived side-effects associated with the use of exogenous hormones, including increased risks of breast cancer, depression, prolonged menstrual cycle, nausea and migraines.[4,5] Many women are also restricted from using estrogen-based hormonal contraceptives due to medical contraindications.[68] These realities strongly underscore the need for convenient non-hormonal contraceptives. Unfortunately, few options are currently available.

An effective non-hormonal contraceptive mechanism already exists in nature: anti-sperm antibodies (ASAs) in infertile women, particularly polyvalent immunoglobulins such as IgA, can arrest highly motile sperm in mucus and prevent sperm from permeating through mucus and reaching the egg. $[9,10]$ At high sperm concentrations, ASA can agglutinate sperm into clusters that are too large to permeate through mucus.[11] At lower sperm concentration, ASA can also immobilize individual spermatozoa in mucus via multiple low affinity Fc-mucin bonds between sperm-bound ASA and mucins.[12,13] Vaginal delivery of ASA that sustains pharmacologically active doses of ASA in the female reproductive tract can make possible both consistent contraception, and rapid reversibility. Indeed, vaginal delivery of sperm agglutinating ASA exhibited considerable contraceptive efficacy in a rabbit model, reducing embryo formation by $95 \%$ in the highly fertile rabbit model.[14] 
Owing to its prevalence at mucosal surfaces and strong agglutination potency due to the diametrically-opposite orientation of the 4 Fab domains, slgA represents an attractive format to develop ASA-based contraceptive.[15] Unfortunately, this topical passive immunocontraception approach has never been advanced in humans, in part because of manufacturing challenges and stability issues with slgA and IgM. In contrast, IgGs are highly stable and easy to produce, making IgG the most common antibody format for development of biologics. We hypothesized we could overcome the instability and production challenges of slgA as well as the limited agglutination potency of IgG by creating IgG molecules with 4 Fabs, identical to slgA. This led us to engineer two tetravalent (i.e. 4 Fabs) sperm-binding IgGs: FabIgG and IgG-Fab, based on linking an additional Fab to either the N- or C- terminus respectively, of a parent IgG using a flexible glycine-serine linker (Figure 1a). We designed our engineered Fab-IgG and IgG-Fab molecules to target a surface glycoprotein antigen, CD52g, a glycoform of CD52 that is unique to the male reproductive tract and present in abundant quantities on the surface of all sperm, but absent in all other tissues and women.[16,17]

Upon transient transfection in Expi293 cells, Fab-lgG and IgG-Fab expressed at comparable levels to the parent IgG (Figure S1a). The parent IgG, Fab-lgG and IgG-Fab all exhibited their expected molecular weights of $150 \mathrm{kDa}, 250 \mathrm{kDa}$ and $250 \mathrm{kDa}$, respectively (Figure 1b and Figure S1b). Surprisingly, while the parent IgG possessed a small fraction of aggregates that is commonly observed with IgGs, the tetravalent IgGs appeared completely homogeneous with no detectable aggregation (Figure 1c). We next evaluated the thermostability of the Fab-IgG and IgG-Fab using Differential Scanning Fluorimetry (DSF). Both constructs exhibited exceptional thermal stability, unfolding only at high temperatures of $\mathrm{Tm}_{1}$ (midpoint of unfolding of $\mathrm{Fab}$ and $\mathrm{C}_{\mathrm{H}} 2$ ) $\geq 71.1^{\circ} \mathrm{C}$ and $\mathrm{Tm}_{2}$ (midpoint of unfolding of $\mathrm{C}_{\mathrm{H}} 3$ ) $\geq 80^{\circ} \mathrm{C}$, comparable to those of the parent IgG (Figure S1c). The incorporation of additional Fab appeared to have a stabilizing effect as $\mathrm{Tm}_{1}$ of Fab-lgG and IgG-Fab is slightly greater than $\mathrm{Tm}_{1}$ of native IgG. We next confirmed the binding of Fab-IgG and IgG-Fab to their sperm antigen using a whole sperm ELISA assay. Both constructs bound comparably to the human sperm as the parent IgG at $0.1 \mu \mathrm{g} / \mathrm{mL}$ (Figure S1d). We visually confirmed sperm agglutination by the parent IgG vs. tetravalent $\lg G$ s at their effective concentrations using scanning electron microscopy (Figure 2).

Progressively motile (PM) sperm, due to their capacity to swim through mucus to reach the egg, is the key sperm fraction responsible for fertilization. We thus assessed the ability of Fab-lgG and IgG-Fab to agglutinate human sperm, using an in vitro sperm escape assay that quantified the number of PM sperm that escaped agglutination when treated with specific antibodies (Abs) vs the sperm washing media 
control determined by Computer Assister Sperm Analysis (CASA).[18,19] The assay was carried out at a concentration of 5 million PM sperm/mL, reflecting typical amounts of PM sperm in fertile males.[20,21] We found that Fab-IgG and IgG-Fab both exhibited markedly greater sperm agglutination potency than parent IgG. The minimal mAb concentrations needed to reduce PM sperm >98\% was reduced from 6.25 $\mu \mathrm{g} / \mathrm{mL}$ for the parent IgG to $1.56 \mu \mathrm{g} / \mathrm{mL}$ for both Fab-lgG and IgG-Fab (Figure 3a). Although both constructs failed to achieve $>98 \%$ agglutination at $0.39 \mu \mathrm{g} / \mathrm{mL}$, they were still able to reduce PM sperm by $>80 \%$ (Figure $3 b$ ). In comparison, the parent IgG at $0.39 \mu \mathrm{g} / \mathrm{mL}$ offered no detectable reduction in PM sperm populations.

For effective contraception, sperm must be stopped in mucus before they can swim through the cervix and access the uterus.[22] This suggests Abs that could agglutinate sperm more quickly should provide more effective contraception. Thus, we next quantified the sperm agglutination kinetics of both constructs using CASA by measuring the fraction of agglutinated and free PM sperm over time immediately after mixing washed sperm with different $m A b s$. At $6.25 \mu \mathrm{g} / \mathrm{mL}$, the parent IgG reduced PM sperm by $\geq 90 \%$ within 90 s in 5 of 6 semen samples; at $1.56 \mu \mathrm{g} / \mathrm{mL}$, the parent failed to do so in all 6 of 6 samples (Figure 3c). In contrast, Fab-IgG and IgG-Fab achieved $\geq 90 \%$ agglutination within 30s in all 6 of 6 samples at $6.25 \mu \mathrm{g} / \mathrm{mL}$, and within $60 \mathrm{~s}$ in 5 of 6 samples at $1.56 \mu \mathrm{g} / \mathrm{mL}$. Notably, the agglutination kinetics of Fab-lgG and IgG-Fab were markedly faster and more complete than the parent IgG at each $A b$ concentrations tested and across all timepoints (Figure 3d).

Earlier works have shown that IgA and IgG Abs can completely immobilize individual spermatozoa in the mucus by crosslinking antibody-bound spermatozoa to mucins; this is commonly referred to as the “shaking phenomenon'.[10] We have previously shown that multiple Fc present on Herpes-bound IgGs can form polyvalent adhesive interactions with cervicovaginal mucus (CVM), resulting in effective immobilization of the virus that in turn blocked vaginal Herpes transmission in mice.[12] Since the Fc region was conserved and identical in both tetravalent IgGs and parent IgG, we hypothesized that Fab-lgG and IgG-Fab constructs will trap individual spermatozoa in mucus similar to native IgG. We evaluated the muco-trapping potencies of the parent and tetravalent IgGs in CVM by multiple particle tracking, using a convolutional neural network to quantify the motion of fluorescently labeled sperm in mAb-treated CVM.[23] Both IgG-Fab and Fab-IgG reduced the fraction of PM sperm to a comparable extent as the parent IgG (Figure 4), indicating that Fc-mediated crosslinking remained unaffected by the addition of Fabs at both $\mathrm{N}$ - and $\mathrm{C}$-terminus. 
Years ago, observations with naturally occurring immune infertility in women motivated the development of contraceptive vaccines.[24-27] Vaccines eliciting ASA offered considerable contraceptive efficacy, but the approach stalled due to unresolved variability in the intensity and duration of the vaccine responses in humans, as well as concerns that active vaccination might lead to permanent infertility.[28] In contrast, the local delivery of ASA to the vagina can overcome the key drawbacks of contraceptive vaccines by sustaining pharmacologically active doses of ASA and making possible both consistent contraception, and rapid reversibility. Unlike small molecule contraceptives, contraceptive mAbs should be exceptionally safe due to the specificity of targeting, particularly when binding to unique epitopes present only on sperm and not on expressed in female tissues. Safety is likely to be further enhanced by topical delivery: mAb delivered to mucosal surfaces such as the vagina are poorly absorbed into the systemic circulation,[29] and the vagina represents a poor immunization inductive site, with limited immune response even when vaccinating with the aid of highly immunostimulatory adjuvants.[30] Topical delivery also substantially reduces the overall mAb dose needed. Given the limited volume of secretions in the female reproductive tract (FRT), typically $\leq 1 \mathrm{~mL}$ in the vagina,[31] relatively high concentration of $\mathrm{mAb}$ locally can be achieved even with very limited total quantities of mAb dosed. In contrast, systemically dosed mAbs must contend with the large blood volume ( $5 \mathrm{~L})$, distribution to non-target tissues, natural catabolic degradation, and limited distribution into the FRT, including the vagina. The reduced quantities of mAb needed to sustain contraceptive levels in the FRT with vaginal delivery should translate to substantially lower amounts of total mAb needed, and consequently cost savings.

Many of the current multivalent Abs are bispecific or trispecific in nature and must content with potential mispairing of light and heavy chains. As a result, many such engineered Ab formats, such as single-chain variable fragment (scFv) or camel-derived nanobodies, involve substantial deviation from natural human $A b$ structure. scFv-based multivalent Ab constructs frequently suffer from low stability, heterogeneous expression, and decreased affinity and specificity stemming from the removal of the $\mathrm{C}_{\mathrm{H}} 1 / \mathrm{C}_{\mathrm{L}}$ interface present in a full-length Fab. The introduction of orthogonal mutations to facilitate heavy and light chain pairing can also substantially reduce $\mathrm{mAb}$ yield or overall stability. These limitations do not apply when generating monospecific multivalent IgGs, which can possess identical and full length human Fabs. Our strategy to covalently link additional Fabs to a parent heavy chain also contrasts from current multimerization strategies based on self-assembly of multiple IgGs based on Fc-mutations [32] or appending an IgM tail-piece, which often suffers poor homogeneity and stability.[33,34] The combination of fully intact human Fabs and covalent linkages likely contributes to the surprising thermal stability, 
homogeneity and bioprocessing ease of the multivalent IgGs developed here. We believe the $\lg G$ multimerization strategy presented here is likely a promising platform for developing mAbs where agglutination represents a critical effector function.

\section{Acknowledgements}

This work was financially supported by the Eshelman Institute of Innovation (S.K.L.); The David and Lucile Packard Foundation (2013-39274; S.K.L); National Institutes of Health under grants R56HD095629 (S.K.L.), U54HD096957 (T.R.M. and S.K.L.), R43HD094454 (T.R.M.) and R44HD097063 (T.R.M.); National Science Foundation (DMR-1810168; S.K.L.); and PhRMA Foundation Graduate Fellowship (B.S.). Special thanks to Dr. Deborah O'Brien for providing the CASA instrument and assistance in setting up the CASA measurements. Special thanks to the UNC Macromolecular Interactions Facility for instruments used in SEC-MALS and DSF studies, and Microscopy Services Laboratory for instruments used in SEM experiments.

\section{Conflict of interest}

S.K.L is the founder of Mucommune, LLC and currently serves as its interim CEO. S.K.L is also the founder of Inhalon Biopharma, Inc, and currently serves as its CSO, Board of Director, and Scientific Advisory Board. S.K.L has equity interests in both Mucommune and Inhalon Biopharma; S.K.L's relationships with Mucommune and Inhalon are subject to certain restrictions under University policy. The terms of these arrangements are managed by UNC-CH in accordance with its conflict of interest policies. T.R.M has equity interests in Inhalon Biopharma. B.S, A.S, T.M.J, T.R.M, and S.K.L are inventors on patents licensed by Mucommune and Inhalon Biopharma. 
Figures with legends

a

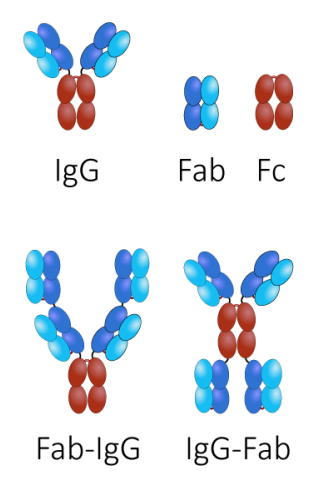

b

Non-Reduced
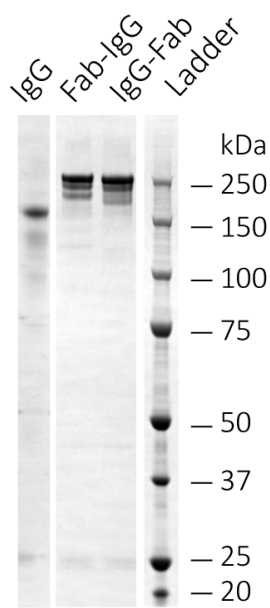

TCEP-Reduced

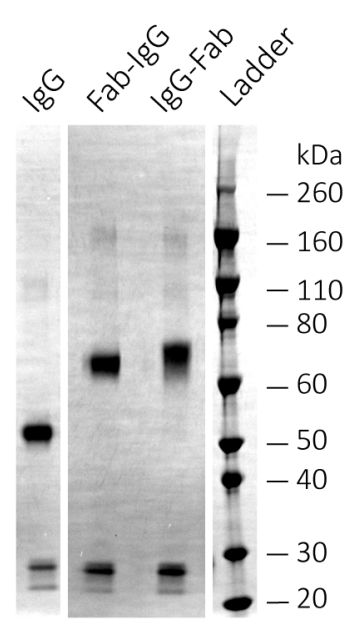

C

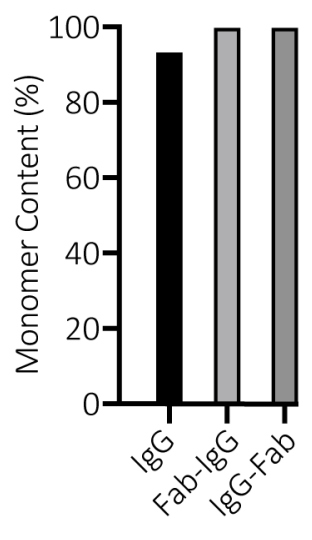

Figure 1. Production and characterization of tetravalent anti-sperm IgG antibodies. (a) Schematic diagrams of anti-sperm IgG, Fab-IgG and IgG-Fab. The additional Fab is linked to the N-terminal or Cterminal of parent IgG using flexible glycine-serine linkers. (b) Non-reducing and reducing SDS-Page analysis of the indicated Abs $(1 \mu \mathrm{g})$ after expression in Expi293 cells and purification by protein A/G chromatography. Non-reducing SDS-Page showcases the total molecular weight of the Abs. Tris (2carboxyethyl) phosphine hydrochloride (TCEP)-mediated reducing SDS-Page displays the molecular weight of individual heavy chain and light chain of Abs. The experiment was repeated independently two times with similar results. The SDS-PAGE image was adjusted for brightness and contrast using Fiji software. (c) Demonstration of the purity and homogeneity of the indicated Abs (50-100 $\mu \mathrm{g}$ ) using Size Exclusion Chromatography with Multiple Angle Light Scattering (SEC-MALS) analysis. Y-axis indicates the total percentage of Abs representing their theoretical molecular weights. 
bioRxiv preprint doi: https://doi.org/10.1101/2020.04.27.064865; this version posted April 29, 2020. The copyright holder for this preprint (which was not certified by peer review) is the author/funder, who has granted bioRxiv a license to display the preprint in perpetuity. It is made available under aCC-BY-NC-ND 4.0 International license.

PBS

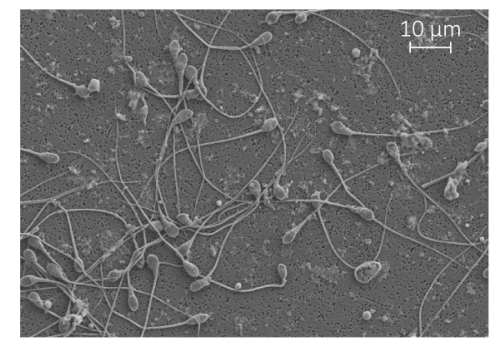

Fab-lgG-1.56 $\mu \mathrm{g} / \mathrm{mL}$

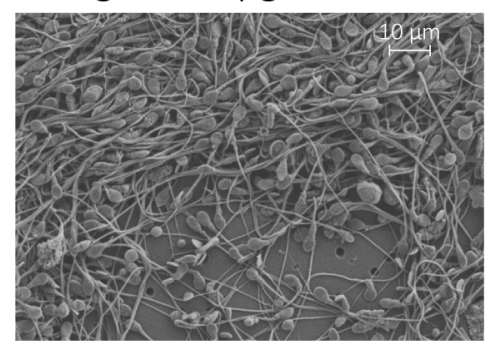

$\operatorname{lgG}-6.25 \mu \mathrm{g} / \mathrm{mL}$

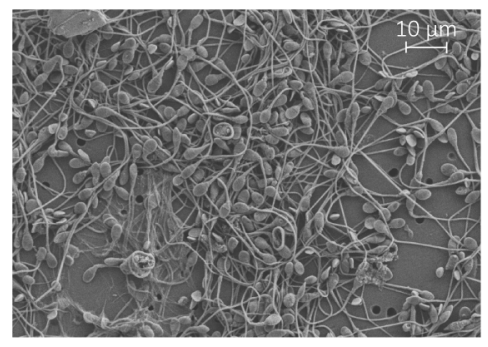

IgG-Fab-1.56 $\mu \mathrm{g} / \mathrm{mL}$

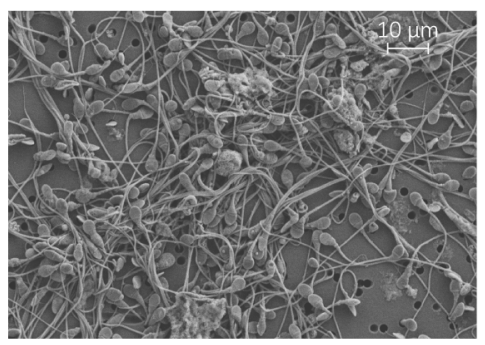

Figure 2. Scanning electron microscopy images of sperm agglutination. 20 million washed sperm were treated with IgG, Fab-IgG and IgG-Fab for 5 min and fixed with 4\% PFA. Images were obtained at 2500X magnification. Scale bar, $10 \mu \mathrm{m}$. 

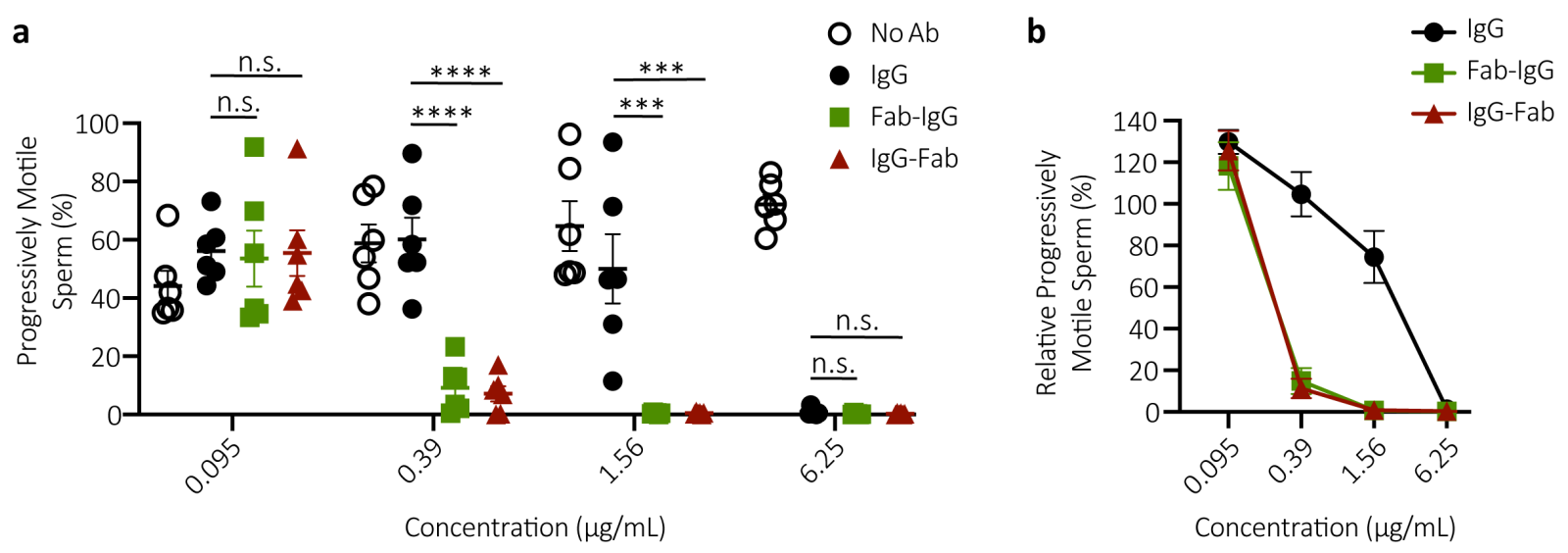

C
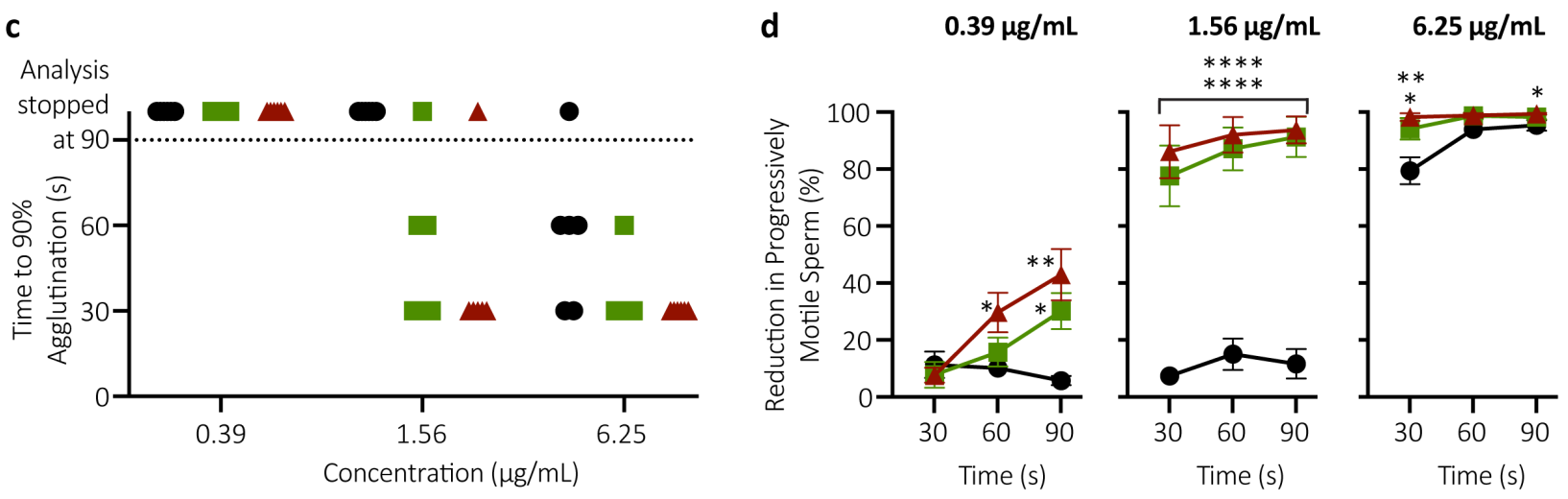

Figure 3. Multimerization markedly enhances the agglutination potency and kinetics of anti-sperm IgG antibodies.

(a) Sperm agglutination potency of the parent IgG, Fab-lgG and IgG-Fab measured by the CASA-based quantification of the percentage of sperm that remains progressively motile (PM) after Ab-treatment compared to pre-treatment condition. (b) The sperm agglutination potency of the Abs normalized to the sperm washing media control. (c) Sperm agglutination kinetics of the parent IgG, Fab-IgG and IgG-Fab measured by the quantification of time required to achieve $90 \%$ agglutination of PM sperm compared to the sperm washing media control. (d) The rate of sperm agglutination determined by the reduction in percentage of PM sperm count at three timepoints after Ab-treatment compared to the sperm washing media control. Purified motile sperm at the final concentration of $5 \times 10^{6} \mathrm{PM}$ sperm $/ \mathrm{mL}$ was used. Data were obtained from $n=6$ independent experiments with at least $n=4$ unique semen donors. Each experiment was performed in duplicates and averaged. P values were calculated using a one-way ANOVA with Dunnett's multiple comparisons test. ${ }^{*} \mathrm{P}<0.05,{ }^{* *} \mathrm{P}<0.01,{ }^{* * *} \mathrm{P}<0.001$ and ${ }^{* * * *} \mathrm{P}<0.0001$. Lines indicate arithmetic mean values and standard error of mean. 


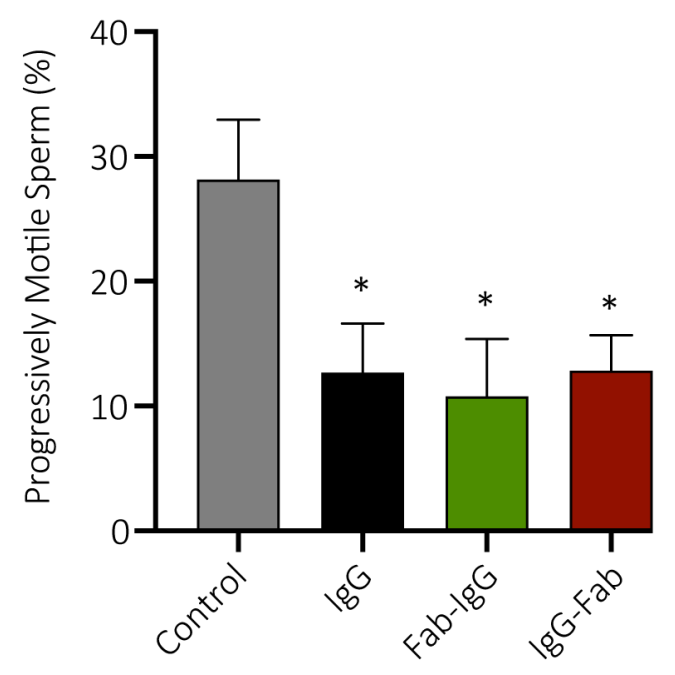

Figure 4. Tetravalent sperm-binding IgG constructs conserve the trapping potency of the parent IgG.

The trapping potency of the indicated Abs measured by quantifying the percentage of fluorescently labeled PM sperm in Ab-treated CVM using neural network tracker analysis software. $25 \mu \mathrm{g} / \mathrm{mL}$ of Abs and purified motile sperm at the final concentration of $5.8 \times 10^{4} \mathrm{PM}$ sperm $/ \mathrm{mL}$ were used. Data were obtained from $n=6$ independent experiments with 6 unique combinations of semen and CVM specimens. $P$ values were calculated using a one-tailed t-test. ${ }^{*} P<0.05$. Lines indicate arithmetic mean values and standard error of mean. 


\section{Materials and methods}

\section{Study design and ethics}

All studies were performed following a protocol approved by the Institutional Review Board of the University of North Carolina at Chapel Hill (IRB-101817). Informed written consent was obtained from all male and female subjects before the collection of any material. Subjects were recruited from the Chapel Hill and Carrboro, NC area in response to mass student emails and posters.

\section{Cloning and production of the parent and tetravalent anti-sperm IgG antibodies}

The variable heavy $\left(V_{H}\right)$ and variable light $\left(V_{L}\right)$ DNA sequences for anti-sperm IgG1 antibody $(A b)$ were obtained from the published sequence of H6-3C4 mAb.[11, 35] For light chain (LC) production, a gene fragment consisting of $V_{L}$ and $C_{L}$ sequences (Integrated DNA Technologies) was cloned into an empty mammalian expression vector (pAH, ThermoFisher Scientific). For parent IgG and Fab-lgG heavy chain (HC) production, $V_{H}$ and $V_{H} / C_{H} 1-\left(G_{4} S\right)_{6}$ linker- $V_{H}$ gene fragments (GeneArt, ThermoFisher Scientific) were respectively cloned into mammalian IgG1 expression vector comprising of only $\mathrm{C}_{\mathrm{H}} 1-\mathrm{C}_{\mathrm{H}} 2-\mathrm{C}_{\mathrm{H}} 3$ DNA sequence. For IgG-Fab $H C$ production, $\left(G_{4} S_{6}{ }_{6}\right.$ linker- $V_{H} / C_{H} 1$ was cloned into the parent IgG expression plasmid. The expression plasmids encoding HC and LC sequences were co-transfected into Expi293F cells using ExpiFectamine ${ }^{\mathrm{TM}} 293$ Transfection reagents (Gibco). For IgG expression, HC and LC plasmid were cotransfected using a 1:1 ratio at $1 \mu \mathrm{g}$ total DNA per $1 \mathrm{~mL}$ of culture. For Fab-lgG and IgG-Fab expression, $\mathrm{HC}$ and LC plasmid were co-transfected using a 1:2 ratio at $1 \mu \mathrm{g}$ total DNA per $1 \mathrm{~mL}$ culture. Transfected Expi293F cells were grown at $37^{\circ} \mathrm{C}$ in a $5 \% \mathrm{CO}_{2}$ incubator and shaken at 125 r.p.m. for 3-5 days. Supernatants were harvested by centrifugation at $12,800 \mathrm{~g}$ for $10 \mathrm{~min}$, passed through $0.22 \mu \mathrm{m}$ filters and purified using standard protein A/G chromatography method. Purified Abs were quantified using absorbance at $280 \mathrm{~nm}$ along with corresponding protein extinction coefficients.

\section{Antibody characterization using SDS-PAGE, SEC-MALS and nanoDSF}

Purified anti-sperm IgG Abs were first assessed for molecular size by SDS-PAGE. Briefly, $1 \mu \mathrm{g}$ of protein was diluted in $3.75 \mu \mathrm{L}$ LDS sample buffer followed by the addition of $11.25 \mu \mathrm{L}$ nuclease-free water. Proteins were then denatured at $70^{\circ} \mathrm{C}$ for $10 \mathrm{~min}$ in a thermocycler. Next, $0.3 \mu \mathrm{L}$ of $0.5 \mathrm{M}$ tris (2carboxyethyl) phosphine (TCEP) was added as a reducing agent to the denatured protein for reduced samples and incubated at room temperature (RT) for 5 min. Bio-Rad Precision Protein Plus Unstained 
Standard and Novex ${ }^{\mathrm{TM}}$ Sharp Pre-stained Protein Standard were used as ladders. After loading the samples, the gel was run for 50 min at a constant voltage of $200 \mathrm{~V}$ and washed 3 times with Milli-Q water. Then, the protein bands were visualized by staining with Imperial Protein Stain (Thermo Scientific) for 1 hr followed by overnight de-staining with Milli-Q water. Image J software (Fiji) was used to adjust the brightness and contrasts of the SDS-PAGE gel for visual purposes.

For SEC-MALS experiment, GE Superdex 200 10/300 column connected to an Agilent FPLC system, a Wyatt DAWN HELEOS II multi-angle light-scattering instrument (Wyatt Technology, Santa Barbara, CA), and a Wyatt T-rEX refractometer were used. The flow rate was maintained at $0.5 \mathrm{~mL} / \mathrm{min}$. The column was equilibrated with 1 X PBS, pH 7.4 containing $200 \mathrm{mg} / \mathrm{L}$ of $\mathrm{NaN}_{3}$ before sample loading. 50-100 $\mu \mathrm{g}$ of each sample was injected onto the column, and the MALS data were collected and analyzed using Wyatt ASTRA software (Ver. 6).

Next, nanoDSF was used to measure the melting $\left(T_{m}\right)$ and aggregation $\left(T_{a g g}\right)$ temperatures of the Abs by performing thermal denaturation experiments at the rate of $1{ }^{\circ} \mathrm{C} / \mathrm{min}$ from $25^{\circ} \mathrm{C}$ to $95^{\circ} \mathrm{C}$. The intrinsic tryptophan fluorescence at $330 \mathrm{~nm}$ and $350 \mathrm{~nm}$ was measured. The melting temperature for each experiment was automatically calculated by Nanotemper PR. Thermcontrol software by plotting the ratiometric measurement of the fluorescent signal against increasing temperature. The aggregation temperature for each experiment was also automatically calculated by Nanotemper PR. Thermcontrol software via the detection of the back-reflection intensity of a light beam that passes the sample.

\section{Collection and processing of semen samples}

Healthy male subjects were asked to refrain from sexual activity for at least $24 \mathrm{hr}$ prior to semen collection. Semen was collected by masturbation into sterile $50 \mathrm{~mL}$ sample cups and incubated for a minimum of 15 min post-ejaculation at RT to allow liquefaction. Semen volume was measured, and the density gradient sperm separation procedure (Irvine Scientific) was used to extract motile sperm from liquefied ejaculates. Briefly, $1.5 \mathrm{~mL}$ of liquified semen was carefully layered over $1.5 \mathrm{~mL}$ of Isolate ${ }^{\circledR}(90 \%$ density gradient medium, Irvine Scientific) at RT, and centrifuged at $300 \mathrm{~g}$ for $20 \mathrm{~min}$. Following centrifugation, the upper layer containing dead cells and seminal plasma was carefully removed without disturbing the motile sperm pellet in the lower layer. The sperm pellet was then washed twice with the sperm washing medium (Irvine Scientific) by centrifugation at $300 \mathrm{~g}$ for $10 \mathrm{~min}$. Finally, the purified motile sperm pellet was resuspended in the sperm washing medium, and an aliquot was taken for determination of sperm count and motility using computer-assisted sperm analysis (CASA). All semen samples used in 
the functional assays exceeded lower reference limits for sperm count $\left(15 \times 10^{6}\right.$ total sperm $\left./ \mathrm{mL}\right)$ and total motility (40\%) as indicated by WHO guidelines.[21]

\section{Sperm count and motility using CASA}

The Hamilton-Thorne computer-assisted sperm analyzer (Hamilton Thorne, Beverly, MA), 12.3 version, was used for the sperm count and motility analysis. For each analysis, $4.4 \mu \mathrm{L}$ of the semen sample was inserted into MicroTool counting chamber slides (Cytonix) and six randomly selected microscopic fields, near the center of the slide, were imaged and analyzed for progressively motile (PM) and nonprogressively motile (NPM) sperm count. The parameters that were assessed by CASA for motility analysis were as follows: average pathway velocity (VAP), the straight-line velocity (VSL), the curvilinear velocity $(\mathrm{VCL})$, the lateral head amplitude (ALH), the beat cross-frequency (BCF), the straightness (STR) and the linearity (LIN). PM sperm were defined as having a minimum of $25 \mu \mathrm{m} / \mathrm{s}$ VAP and $80 \%$ of STR. The complete parameters of the Hamilton-Thorne Ceros 12.3 software are listed in Table S1.[18,19]

\section{Whole sperm ELISA}

Briefly, half-area polystyrene plates (CLS3690, Corning) were coated with $2 \times 10^{5}$ sperm per well in $50 \mu \mathrm{L}$ of $\mathrm{NaHCO}_{3}$ buffer ( $\mathrm{pH}$ 9.6). After overnight incubation at $4^{\circ} \mathrm{C}$, the plates were centrifuged at the speed of $300 \mathrm{~g}$ for $20 \mathrm{~min}$. The supernatant was discarded, and the plates were air-dried for $1 \mathrm{hr}$ at $45^{\circ} \mathrm{C}$. The plates were washed once with 1 X PBS. $100 \mu \mathrm{L}$ of $5 \%$ milk was incubated at RT for $1 \mathrm{hr}$ to prevent non-specific binding of Abs to the microwells. The serial dilution of mAbs in $1 \%$ milk was added to the microwells and incubated overnight at $4^{\circ} \mathrm{C}$. Motavizumab, a mAb against the respiratory syncytial virus, was constructed and expressed in the laboratory by accessing the published sequence and used as a negative control for this assay. After primary incubation, the plates were washed three times using $1 \mathrm{X}$ PBS. Then, the secondary Ab, goat anti-human IgG $\mathrm{F}\left(\mathrm{ab} \mathrm{b}^{\prime}\right)_{2}$ Ab HRP-conjugated (1:10,000 dilutions in 1\% milk, 209-1304, Rockland Inc.) was added to the wells and incubated for $1 \mathrm{hr}$ at RT. The washing procedure was repeated and $50 \mu \mathrm{L}$ of the buffer containing substrate (1-Step Ultra TMB ELISA Substrate, Thermo Scientific) was added to develop the colorimetric reaction for $15 \mathrm{~min}$. The reaction was quenched using $50 \mu \mathrm{L}$ of $2 \mathrm{~N}$ $\mathrm{H}_{2} \mathrm{SO}_{4}$, and the absorbance at $450 \mathrm{~nm}$ (signal) and $570 \mathrm{~nm}$ (background) was measured using SpectraMax M2 Microplate Reader (Molecular Devices, San Jose, CA). Each experiment was done with samples in triplicates and repeated two times as a measure of assay variability. 


\section{Scanning electron microscopy}

Briefly, $20 \times 10^{6}$ washed sperm was centrifuged at $300 \mathrm{~g}$ for $10 \mathrm{~min}$ and the supernatant was discarded without disturbing the sperm pellet. Then, $200 \mu \mathrm{L}$ of Abs or 1X PBS was added to the sperm pellet, mixed by pipetting and incubated for 5 mins using an end-over-end rotator. Next, $200 \mu \mathrm{L}$ of 4\% PFA was added to the Ab-sperm solution and incubated for 10 min using an end-over-end rotator. $50 \mu \mathrm{L}$ of fixed sperm samples was filtered and washed through membrane filters (10562, K04CP02500, Osmonics) using 0.15 M Sodium Phosphate buffer. The samples were then dehydrated in a graded series of alcohol, transferred to a plate with the transitional solvent, Hexamethyldisilazane (Electron Microscopy Sciences) and allowed to dry after one exchange. Next, filters were adhered to aluminum stubs with carbon adhesives and samples were sputter-coated with gold-palladium alloy (Au:Pd 60:40 ratio, 91112, Ted Pella Inc.) to a thickness of $3 \mathrm{~nm}$ using Cressington Sputter Coater $208 \mathrm{hr}$. Six random images were acquired for each sample using a Zeiss Supra 25 FESEM (Carl Zeiss Microscopy, White Plains, NY) with an SE2 Electron detector at 2500X magnification.

\section{Sperm escape assay}

Briefly, $40 \mu \mathrm{L}$ aliquots of purified motile sperm $\left(10 \times 10^{6} \mathrm{PM}\right.$ sperm $\left./ \mathrm{mL}\right)$ was transferred to individual 0.2 $\mathrm{mL}$ PCR tubes. Sperm count and motility were performed again on each $40 \mu \mathrm{L}$ aliquot using CASA. This count serves as the original (untreated) concentration of sperm for evaluating the agglutination potencies of respective Ab constructs. Following CASA, $30 \mu \mathrm{L}$ of purified motile sperm was mixed into $0.2 \mathrm{~mL}$ PCR tubes containing $30 \mu \mathrm{L}$ of Abs or sperm washing medium control. The tubes were then fixed at $45^{\circ}$ angles for $5 \mathrm{~min}$ at RT. Following this incubation period, $4.4 \mu \mathrm{L}$ was extracted from the top layer of the mixture with minimal perturbation of the tube and transferred to the CASA instrument to quantify the number of PM sperm. The percentage of the PM sperm that escaped agglutination was computed by dividing the sperm count obtained after treatment with $A b$ constructs by the original untreated sperm count in each respective tube, correcting for the 2 -fold dilution with $\mathrm{Ab}$. Each experimental condition was evaluated in duplicates on each semen specimen, and the average from the two experiments was used in the analysis. Data represents 6 independent experiments with at least $n=4$ unique semen samples. $P$ values were calculated using a one-way ANOVA with Dunnett's multiple comparisons test.

\section{Agglutination kinetics assay}


Briefly, $4.4 \mu \mathrm{L}$ of purified motile sperm $\left(10 \times 10^{6} \mathrm{PM}\right.$ sperm $\left./ \mathrm{mL}\right)$ was added to $4.4 \mu \mathrm{L}$ of Ab constructs in $0.2 \mathrm{~mL}$ PCR tubes followed by gentle mixing. Immediately, a timer was started and $4.4 \mu \mathrm{L}$ of the mixture was transferred to chamber slides. The center field of the slides was then imaged and analyzed by CASA instrument every $30 \mathrm{~s}$ up to $90 \mathrm{~s}$. The reduction in the percentage of PM sperm at each time point was computed by normalizing the PM sperm count obtained after Ab treatment to the PM sperm count obtained after control treatment with sperm washing medium. Each experimental condition was evaluated in duplicates on each semen specimen, and the average from the two experiments was used in the analysis. Data represents 6 independent experiments with at least $n=4$ unique semen samples. $P$ values were calculated using a one-way ANOVA with Dunnett's multiple comparisons test.

\section{Fluorescent labeling of sperm}

Purified motile sperm were fluorescently labeled using Live/Dead Sperm Viability Kit (Invitrogen, Thermofisher Scientific). SYBR 14 dye, a membrane-permeant nucleic acid stain, stained the live sperm while propidium iodide (PI), a membrane impermeant nucleic acid stain, stained the dead sperm. SYBR14 and PI dye were added to $1 \mathrm{~mL}$ of washed sperm resulting in final SYBR 14 and PI concentration of 200 $\mathrm{nM}$ and $12 \mu \mathrm{M}$ respectively and incubated for $10 \mathrm{~min}$ at $36^{\circ} \mathrm{C}$. The sperm-dye solution was washed twice using the sperm washing medium to remove unbound fluorophores by centrifuging at $300 \mathrm{~g}$ for $10 \mathrm{~min}$. Next, the labeled motile sperm pellet was resuspended in the sperm washing medium, and an aliquot was taken for determination of sperm count and motility using CASA.

\section{CVM collection and processing}

CVM was collected as previously described.[12] Briefly, undiluted CVM secretions, averaging 0.5 g per sample, were obtained from women of reproductive age, ranging from 20 to 44 years old, by using a selfsampling menstrual collection device (Instead Softcup). Participants inserted the device into the vagina for at least $30 \mathrm{~s}$, removed it, and placed it into a $50 \mathrm{~mL}$ centrifuge tube for collection. Samples were collected at various times throughout the menstrual cycle, and the cycle phase was estimated based on the last menstrual period date normalized to a 28-day cycle. Samples that were non-uniform in color or consistency were discarded. Donors stated they had not used vaginal products nor participated in unprotected intercourse within 3 days before donating. All samples had $\mathrm{pH}<4.5$. 


\section{Multiple particle tracking of fluorescently labeled sperm in mucus}

Multiple particle tracking was performed as previously described.[12] Briefly, fresh CVM was diluted three-fold using sperm washing medium to mimic the dilution and neutralization of CVM by alkaline seminal fluid in humans and titrated to $\mathrm{pH}$ 6.8-7.1 using small volumes of $3 \mathrm{~N} \mathrm{NaOH}$. Next, $4 \mu \mathrm{L}$ of Abs or control (anti-RSV IgG1) was added to $60 \mu \mathrm{L}$ of diluted and pH-adjusted CVM and mixed well in a CultureWell ${ }^{\mathrm{TM}}$ chamber slides (Invitrogen, ThermoFisher Scientific) followed by mixing of $4 \mu \mathrm{L}$ of $1 \times 10^{6}$ fluorescently labeled PM sperm $/ \mathrm{mL}$. Chamber slides were incubated for $5 \mathrm{~min}$ at RT. Then, translational motions of the fluorescently labeled sperm were recorded using an electron-multiplying charge-coupleddevice camera (Evolve 512; Photometrics, Tucson, AZ) mounted on an inverted epifluorescence microscope (AxioObserver D1; Zeiss, Thornwood, NY) equipped with an Alpha Plan-Apo 20/0.4 objective, environmental (temperature and $\mathrm{CO} 2$ ) control chamber, and light-emitting diode (LED) light source (Lumencor Light Engine DAPI/GFP/543/623/690). 15 videos (512 ×512 pixels, 16-bit image depth) were captured for each Ab condition with MetaMorph imaging software (Molecular Devices, Sunnyvale, CA) at a temporal resolution of $66.7 \mathrm{~ms}$ and spatial resolution of $50 \mathrm{~nm}$ (nominal pixel resolution, $0.78 \mu \mathrm{m} / \mathrm{pixel}$ ) for $10 \mathrm{~s}$. Next, the acquired videos were run through a neural network tracking software modified with standard sperm motility parameters to determine the percentage of PM sperm.[23] Data represents 6 independent experiments, each using a unique combination of CVM and semen specimens. P values were calculated using a one-tailed t-test.

\section{Statistical analysis}

All analyses were performed using GraphPad Prism 8 software. For multiple group comparisons, P values were calculated using a one-way ANOVA with Dunnett's multiple comparisons tests. The comparison between control- and anti-sperm Ab-treated fluorescent PM sperm was performed using a one-tailed ttest. In all analyses, $\alpha=0.05$ for statistical significance. All data are presented as the mean \pm standard error of the mean. 


\section{References}

1. Bearak, J., Popinchalk, A., Alkema, L. \& Sedgh, G. Global, regional, and subregional trends in unintended pregnancy and its outcomes from 1990 to 2014: estimates from a Bayesian hierarchical model Jonathan. Lancet Glob Heal. 6, 380-389 (2018).

2. Finer, L. B. \& Henshaw, S. K. Disparities in Rates of Unintended Pregnancy In the United States, 1994 and 2001. Perspect. Sex. Reprod. Health 38, 90-96 (2006).

3. Finer, L. B. \& Zolna, M. R. Declines in Unintended Pregnancy in the United States, 2008-2011. N. Engl. J. Med. 374, 843-852 (2016).

4. Skouby, S. O. Contraceptive use and behavior in the 21st century: A comprehensive study across five European countries. Eur. J. Contracept. Reprod. Heal. Care 9, 57-68 (2004).

5. Brynhildsen, J. Combined hormonal contraceptives : prescribing patterns, compliance, and benefits versus risks. Ther. Adv. Drug Saf. 5, 201-213 (2014).

6. Grossman, D. et al. Contraindications to combined oral contraceptives among over-the-counter compared with prescription users. Obstet. Gynecol. 117, 558-565 (2011).

7. Beck, W. W. Complications and Contraindications of Oral Contraception. Clinical Obstetrics and Gynecology 24, 893-902 (1981).

8. Roos-Hesselink, J. W. et al. Contraception and cardiovascular disease. Eur. Heart J. 36, 1728-1734 (2015).

9. Kremer, J. \& Jager, S. The Sperm-Cervical Mucus Contact Test: A Preliminary Report. Fertil. Steril. 27, 335-340 (1976).

10. Clarke, G. N. Induction of the Shaking Phenomenon by IgA Class Antispermatozoal Antibodies From Serum. Am. J. Reprod. Immunol. Microbiol. 9, 12-14 (1985).

11. Isojima, S. et al. Establishment and characterization of a human hybridoma secreting monoclonal antibody with high titers of sperm immobilizing and agglutinating activities against human seminal plasma. J. Reprod. Immunol. 10, 67-78 (1987).

12. Wang, Y. Y. et al. IgG in cervicovaginal mucus traps HSV and prevents vaginal Herpes infections. Mucosal Immunol. 7, 1036-1044 (2014).

13. Wang, Y. Y., Harit, D. \& Subramani, D. B. Influenza-binding antibodies immobilise influenza viruses in fresh human airway mucus. Eur Res J 49, 1-4 (2017).

14. Castle, P. E., Whaley, K. J., Hoen, T. E., Moench, T. R. \& Cone, R. A. Contraceptive Effect of SpermAgglutinating Monoclonal Antibodies in Rabbits. Biol. Reprod. 56, 153-159 (1997).

15. Woof, J. M. \& Ken, M. A. The function of immunoglobulin A in immunity. J. Pathol. 208, 270-282 
(2006).

16. Diekman, A. B. et al. N-linked glycan of a sperm CD52 glycoform associated with human infertility. FASEB J. 13, 1303-1313 (1999).

17. Norton, E. J. et al. A male genital tract-specific carbohydrate epitope on human CD52: Implications for immunocontraception. Tissue Antigens 60, 354-364 (2002).

18. Mitra, A., Richardson, R. T. \& O’Rand, M. G. Analysis of Recombinant Human Semenogelin as an Inhibitor of Human Sperm Motility. Biol. Reprod. 82, 489-496 (2010).

19. Hirano, Y. et al. Relationships between sperm motility characteristics assessed by the computeraided sperm analysis (CASA) and fertilization rates in vitro. J. Assist. Reprod. Genet. 18, 213-218 (2001).

20. Sharpe, R. M. Sperm counts and fertility in men: A rocky road ahead. Science \& Society Series on Sex and Science. EMBO Rep. 13, 398-403 (2012).

21. Organization, W. H. Examination and processing of human semen. in World Health Fifth Edit, 1271 (2010).

22. Fordney Settlage, D. S., Motoshima, M. \& Tredway, D. R. Sperm Transport from the External Cervical Os to the Fallopian Tubes in Women: A Time and Quantitation Study. Fertil. Steril. 24, 655-661 (1973).

23. Newby, J. M., Schaefer, A. M., Lee, P. T., Forest, M. G. \& Lai, S. K. Convolutional neural networks automate detection for tracking of submicron-scale particles in 2D and 3D. Proc. Natl. Acad. Sci. U. S. A. 115, 9026-9031 (2018).

24. Naz, R. K. \& Zhu, X. Recombinant Fertilization Antigen-1 Causes a Contraceptive Effect in Actively Immunized Mice. Biol. Reprod. 59, 1095-1100 (1998).

25. Naz, R. K. \& Chauhan, S. C. Human Sperm-Specific Peptide Vaccine That Causes Long-Term Reversible Contraception. Biol. Reprod. 67, 674-680 (2002).

26. Naz, R. K. Immunocontraceptive Effect of Izumo and Enhancement by Combination Vaccination. Mol. Reprod. Dev. 344, 336-344 (2008).

27. Primakoff, P., Lathrop, W., Woolman, L., Cowan, A. \& Myles, D. Fully effective contraception in male and female guinea pigs immunized with the sperm protein $\mathrm{PH}-20$. Nature 335, 543-546 (1988).

28. Naz, R. K. Vaccine for contraception targeting sperm. Immunol. Rev. 171, 193-202 (1999).

29. Hussain, A. \& Ahsan, F. The vagina as a route for systemic drug delivery. J. Control. Release 103, 301-313 (2005). 
30. Zeitlin, L., Cone, R. A. \& Whaley, K. J. Using Monoclonal Antibodies to Prevent Mucosal Transmission of Epidemic Infectious Diseases. Emerg. Infect. Dis. 5, 54-64 (1999).

31. Owen, D. H. \& Katz, D. F. A vaginal fluid simulant. Contraception 59, 91-95 (1999).

32. Diebolder, C. A. et al. Complement Is Activated by IgG Hexamers Assembled at the Cell Surface. Science 343, 1260-1263 (2014).

33. Sorensen, V. et al. Effect of the IgM and IgA secretory tailpieces on polymerization and secretion of IgM and IgG. J. Immunol. 156, 2858-2865 (1996).

34. Teye, K. et al. Multimerization is required for antigen binding activity of an engineered IgM/IgG chimeric antibody recognizing a skin-related antigen. Sci. Rep. 7, 1-12 (2017).

35. Komori S, Yamasaki N, Shigeta M, Isojima S, Takeshi W. Production of heavy-chain class-switch variants of human monoclonal antibody by recombinant DNA technology. Clin. Exp. Immunol. 71, 508-16 (1988).

36. Wu H. et al. Development of Motavizumab, an Ultra-potent Antibody for the Prevention of Respiratory Syncytial Virus Infection in the Upper and Lower Respiratory Tract. J Mol. Biol. 368, 652-65 (2007). 